\title{
Why Rating is not Enough: A Study on Online Reputation Systems
}

\author{
Rehab Alnemr \\ Hasso Plattner Institute \\ Potsdam University \\ Germany \\ rehab.alnemr@hpi.uni-potsdam.de
}

\author{
Christoph Meinel \\ Hasso Plattner Institute \\ Potsdam University \\ Germany \\ meinel@hpi.uni-potsdam.de
}

\begin{abstract}
For several years online reputation systems have been evolving in an attempt to mimic their real-world counterparts. Rating in online communities comes in many forms such as numbers, stars, scales, etc.. The underlying aggregation or computation methods varies according to the community's information sources and perception of reputation. However, the notion of reputation is a complex one that represented as a simple form of property-rating or a vector of ratings, strips it from its original concept and postulation. It does not also facilitate the derivation of meaningful conclusions from it. In this paper, we present studies we designed to examine how users perceive reputation as well as what reputation information being used and whether rating is enough to convey an entity's reputation. These studies are analyzed and discussed based on the results. Moreover, we show how our reputation ontology - that is designed to facilitate reputation knowledge transfer on the communication level- is used in a simple interface of multiple ratings. The model is based on semantic web technologies.
\end{abstract}

Index Terms-Reputation, Rating, Online Reviews, Collaboration

\section{INTRODUCTION}

Reputation is used as one of the approaches to establish trust between unrelated parties in a community. Online reputation systems mainly depend on user collaboration and knowledge sharing. If the users share less, the reputation values will have insignificant meaning because there is not enough information to construct a credible and meaningful trust. Reputation systems sometimes provide an incentive not only to collaborate but also to encourage constructive behavior. However, this open collaboration results in a challenge of what is being shared, how it is being shared, and the common versus the intended meaning of this information.

Most of the existing reputation-based systems lack the connection between general reputation and its context. One can trust a physician to treat him but not to handle his financial transaction. There are some issues that arise in reputation systems due to the design and implementation of the current reputation models. These systems were designed as closed domains, where each one has its own data entry, enquiry, representation and interaction styles, interpretation, computation of reputation values, limited information sources, etc.. [4]

Some of the reputation values online are easy to interpret in terms of what are the reputation attributes involved in the process. Reputation values in Wikipedia, for instance, are explicitly for the quality of the content. Reputation is mainly calculated according to the extent to which the content has been revised. Other reputation systems however, have a problem of rating interpretations i.e. what does the rating mean, its scope, and what are the reputation attributes involved in the ratings.

The goal of our work is to show how reputation representations -in several domains- lack efficient embedment of information. But in order to work on a model that captures the true notion of reputation, we needed to understand how users perceive it first. Though our focus is on different domains, this can only be done by testing user interactions with online reputation systems. Starting by exploring the literature and how researchers analyze reputation attributes and our own observations, we formed two hypothesis. Several user studies start by formulating the hypothesis [13] then design the study to examine whether the results support these hypothesis. The hypothesis are:

Hypothesis 1: In a scenario where a service consumer is to decide on a service provider based on a specific set of preferences, the consumer needs more information than ratings to take his/her decision

Hypothesis 2: In a scenario where a service consumer is rating a service provider or selecting a provider based on his rating, overall rating is not enough to convey an entity's reputation or to describe the reason behind the rating

Based on these hypothesis, we setup an online survey and an experiment. In the online survey, we asked the users several questions designed to capture how they perceive online ratings and what pieces of information used in their decision of buying a product or selecting a service provider. The chocolate online store experiment described in III-B was designed to capture real world ratings data and examine the variation of ratings' reasons.

The paper is organized as following: in section II we define some relevant terminologies, show categories of online reputation systems, general steps to construct one, and discuss problems in current systems. Section III explains our user studies and section IV discuss our analysis. In section V we conclude the paper and discuss future work. 


\section{REPUTATION SYSTEMS}

A reputation model describes all of the reputation statements, events, and processes for a particular context. This context is the relevant category for a specific reputation. In the literature, reputation is defined as an expectation about an entity's behavior based on information about or observations of its past behavior [2]. In the business world, Balmer [8] defines two characteristics for corporate reputation: it evolves through time and is based on what the organization has done and how it has behaved.

We distinguish between: reputation ontology, reputation system, model, or framework, reputation engine or mechanism, and reputation architecture. A reputation ontology describes the notion of reputation and the relations to the concepts that compose it, while a reputation system, model, or framework describes the collection, distribution, and aggregation of reputation information. A reputation computation engine or mechanism is one of the modules in a reputation system which shows how reputation value(s) are calculated. A reputation architecture is a set of protocols that determines how reputation values are communicated between the participants in a reputation system.[5] A reputation context or criterion is defined as a characteristic, a property, or a measurement by which an entity is judged or evaluated in a certain context. Sometimes it is called reputation attribute or quality attribute and in general also it is called reputation scope which defines the general category in which a reputation was created i.e. for e-Market, for web services.

There are extensive studies about reputation systems that discuss not only the current commercial ones but also proposed approaches from academia. For example, studies done by Jøsang in [12] and Sabater in [14], provide an exhaustive view of what's out there in the reputation community. Commercial applications implementing trust and reputation mechanisms use relatively simple schemes than those proposed by research papers.

Online reputation systems are the biggest and most obvious examples of reputation systems. They can be categorized [7] by characteristics as:

1) The subject of rating

2) The providers of the ratings (open to the public or restricted)

3) The business model (revenue derived from an associated online auction, advertising, or a public service)

4) Relative Reviews (whether users ratings are relative to the attributes of the rating)

The subject of the rating varies from rating individuals such as Allexperts.com, eBay or Amazon, business such as BizRate.com, to articles or posting such as in Kuro5hin.org, Slashdot.com, and products and services such as Epinions.com, Amazon. Reputation systems can also be categorized based on the common features and properties of the online communities as:

1) E-market places e.g. eBay
2) Opinions and activity sharing sites e.g. Epinions, Del.icio.us, LastFm

3) Business/Jobs network sites eg. Linkedin.com \& Ryze.com

4) Social/entertainment sites eg. Friendster.com \& Facebook

5) News site eg. Kuroshin.org, Slashdot, \& Zdnet

6) The Web/Semantic Web as for anyone who publish anything in a decentralized way

7) P2P networks where peer clients share opinions about other peers.

Table I shows the rating methods and rating levels from a study we conducted on a several reputation systems. [3] Each System use different kind of rating/raking to evaluate an entity's reputation. In online reputation communities, trust and reputation (or rating at this point) is represented numerically or graphically using bars and stars, karma, or in natural language (i.e. good, bad). The range of possible values for a trust level and the meaning of these values varies according to each system.

A collections of online stores are sometimes known as virtual shopping malls or online marketplaces. Online marketplaces, also known as e-markets, are virtual online markets where individuals and organizations act as buyers and sellers to carry out business to business transactions over the internet. For the buyers, it is a convenient way to compare prices and products from a centralized source. There are several types of these markets such as transactional site with one responsible provider (i.e. Amazon), e-markets with multiple providers (i.e. e-bay).

E-markets use reputation to manage trust between service providers and consumers. In this domain, users collaboration is essential i.e. If the users refrain from sharing their opinions and reviews of a product, the reputation system will seize to exist. Also, if the users share less, the reputation values will have insignificant meaning because there is not enough information to construct a credible and meaningful trust. The problem with these markets, however, is not sharing as what is being shared. The rest of this section shows an example of feedback scenarios and in subsection II-A we discuss this problem.

A typical buying or purchasing scenario in an e-market is that the consumer specify which product he/she needs, navigates the website to locate it, and is presented with several options to select from. In a single provider e-market, where one organization is responsible for the transactions (i.e. Amazon), the consumer is presented with multiple products with different brands and sometimes with variations in their features. In a multiple providers e-market, where sellers and buyers are on the same level (customer-to-customer a.k.a C2C) and the market is responsible for providing the platform that brings them together (i.e. eBay), the consumer is presented with multiple products from several sellers and also with variations in products features.

The reputation of the product and the seller plays a critical role on the decision process of which product to buy and from 
TABLE I

Rating Methods in OnLine Societies

\begin{tabular}{|c|c|c|c|c|c|c|c|}
\hline$\ldots$ & 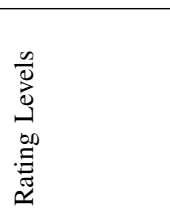 & 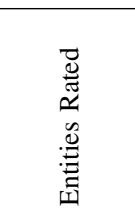 & 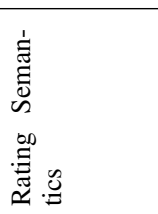 & 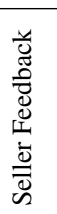 & 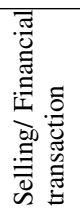 & 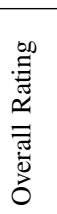 & 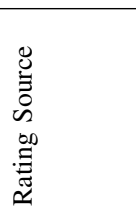 \\
\hline Amazon & 5 & $\begin{array}{c}\text { Sellers } \\
\text { Products } \\
\text {-ve: } 2 \text { or } 1 \\
\text { Reviews }\end{array}$ & $\begin{array}{l}\text { +ve : } 5 \text { or } 4 \\
\text { Neutral: } 3 \\
\text { Hated it: } 1 \\
\text { Loved it: } 5 \\
\end{array}$ & No & Yes & Yes & $\begin{array}{l}\text { Customers } \\
\text { Spotlight }\end{array}$ \\
\hline eBay & $\begin{array}{l}\text { Starts at } 3 \text { till } \\
10\end{array}$ & Members & & Yes & Yes & Yes & Members \\
\hline Epinions & 4 & $\begin{array}{c}\text { Sellers } \\
\text { Products } \\
\\
\text { Reviews } \\
\text { Reviewer } \\
\end{array}$ & $\begin{array}{l}\text { Excellent: } 5 \\
\text { Poor: } 1\end{array}$ & & No & Yes & $\begin{array}{l}\text { Trusted } \\
\text { Agents } \\
\text { Customers }\end{array}$ \\
\hline BizRate & $\begin{array}{l}5 \\
4\end{array}$ & $\begin{array}{l}\text { Products } \\
\text { Merchants } \\
\text { Reviews }\end{array}$ & & No & No & No & $\begin{array}{l}\text { Customers } \\
\text { Trusted } \\
\text { Agents }\end{array}$ \\
\hline Yahoo & 2 & $\begin{array}{c}\text { Sellers } \\
\text { Products } \\
\text { Reviews }\end{array}$ & $\begin{array}{l}\text { Excellent: } 5 \\
\text { Poor:1 } \\
\text { Helpful } \\
\text { Not Helpful }\end{array}$ & Yes & Yes & Yes & Members \\
\hline
\end{tabular}

whom. In Amazon, for instance, the rating system is 1 to 5 stars, with 5 stars being the best. After buying, the consumer is asked to give his feedback in two ways: a) by giving a star ratings and b) by answering a seller feedback questions with an option of leaving a comment. There is no obvious distinction -at the rating page- of what exactly being rated or reviewed. Only at the description page (i.e. information page stating the policies) the user can find the distinction: the star rating is an overall rating of the product, while the detailed review page is for the buying experience which includes reviewing the seller (i.e. order fulfillment, customer service, correct item description). Furthermore, there is a statement in the information page warning the users that: "If your comments include any of the following, your feedback is subject to removal: Product reviews: It is more appropriate to review product on the product detail page....Customers reviews are for products".

\section{A. Problems in the Current Representations and Interfaces}

In the previous section, we briefly discussed the problem of mis-communicating what is actually being reviewed when rating an e-market participant. In our user study, we asked the user to differentiate between the 5 star rating and the reviews and $65 \%$ of them could not locate the difference. Most of the study participants voted that both the rating and the reviews are for product quality while $35 \%$ of them voted that it is for customer service. In this scenario, there are three reputation attributes that should be distinguished: product quality, seller reputation, and customer service. All of these attributes are related to seller's reputation while only the product quality is related to the product reputation.
Another scenario, where the vagueness of the rating is obvious, is when a user is rating a book. The problem is: is the rating for the book itself (i.e. the user liked what he read) or the quality of the book (i.e. was new and good printing) or the service provided by Amazon for example (i.e. offering the book, price, delivery, payment method, etc.)? Rating should differentiate between rating the service and rating the product. Also it should take a different form if the domain is "Multiple Products eMarket" (i.e. when rating Amazon service) or "One Provider eMarket" (i.e. when the rating of the provider includes the quality of the product).

Such problem raised legal hassles. An interesting study about legal challenges that face online reputation systems was conducted in [10]. In this study, the authors explore legal cases against reputation systems as eBay (California, Grace vs.eBay) and Amazon (cases in UK and USA). Mostly, the main reason for these cases is rating ambiguity. Users misreport their ratings in a way that influences negatively the entity being rated and does not correspond to the rating attributes. For example, a seller of second hand books was given bad ratings because a book was not good or too long though the book was in a very good condition which is what matters for rating a used-books seller. Legally, systems like eBay holds no responsibility for users who are expressing their taste. What is important from the legal perspective is the distinction between "expressions of fact" and "opinion". In spite of the fact that eBay instituted limited assurance coverage- Standard Purchase Protection Program - the problem still exists and growing. What these systems need is specific rating attributes categorized and semantically defined. The less 
vague the rating, the fewer legal issues arise.

In general, reputation systems have also other issues some of which are:

- Excluding the context from the reputation value because most representation and exchange format has no embedded information about the context in which reputation was earned. Since context is not usually included in a reputation query, it is assumed that the implicit context is the domain of the reputation system (e.g. rate the seller for this purchase transaction) resulting in a too general query. In online markets, for instance, a consumer rates a seller generally for a trading/purchase transaction, leaving the details to be written in a natural language review.

- Difficulty in mapping between reputation values due to difference in perceptions

- Incorrect modeling and variance in calculations and interpretations because in spite of the wide variety of computation models, most of them do not reflect the real cognitive nature of reputation as they do not represent all the parameters that affect it.

- No portability or interoperability of reputation information because it is hard to exchange the knowledge when the semantics are not considered in the calculation or the representation of reputation. Reputation interoperability can solve problems such as the cold start problem.

These issues explain why it's hard to asses and exchange reputation especially between e-markets due to the difference in perception, calculation, interpretation, but most of all because the given reputation is an overall one that does not reflect the related contexts in which it is earned. These contexts can vary from the category it is earned (i.e. a selling transaction) to the quality aspects of one transaction (i.e. different quality criteria or attributes). [4]

\section{B. Steps to Construct a Reputation Management System}

Based on observations and literature, we explain here how to create a reputation system. When creating -or integrating a reputation system for a business domain, there are general steps to go through. They are:

1) Clearly define why reputation is needed- based on the system focus or the domain purpose- and how it will be used, i.e. to encourage participants' contributions, to help in an entity's selection, enhance quality of products or services, etc.

2) Identify the system participants and entities that composes the system i.e. users, agents, services, products, transactions, network nodes

3) Determine which identity system is used i.e. how would you identify each entity

4) Establish who will have reputation from the previously identified entitys

5) Define the type of reputation associated with each kind or type of participants, i.e. ratings, levels, points, etc.

6) Deduce the criteria that compose the reputation of each entity, i.e. delivery, product quality, and prices for a service provider in an e-market, or service availability, quality, and speed for a web service

7) Ascertain what are the information needed to establish an entity's reputation and how it will be extracted from the system or the domain, i.e. collecting algorithm for each reputation criterion

8) Select a reputation computation function or algorithm that best suits the domain and specific to each crietrion

9) Decide how each reputation is represented if the domain had a user interface

10) Define reputation statements (i.e. the action of rating), reputation responses, and reputation queries

11) Decide access rights for the reputation values, i.e. who will see what

12) Resolve the related reputation factors in the domain, i.e. can we define and describe the factors affecting reputation? does the reputation of the source (reputee) affect the calculation of reputation?

13) Decide how reputation is going to be affected by time These steps are general ones and the list is not exhaustive but they act as general guidelines for constructing a reputation system. However, specialization measures are taken to suit the business domain.

\section{USER STUDIES}

Although our work is focusing on reputation systems in general (not only on online reputation systems), we conduct the following studies on the user perception of online reputation especially in e-market because it is the only tangible reputation the user sees online. In this section we show a user study on how users perceive online ratings, and an experiment to prove that single rating is not enough to represent reputation. To elaborate on the hypothesis that users use more information in their buying decision (hypothesis 1), in the rest of the paper we use three reputation attributes as test-attributes: price, quality, and delivery. The idea is to test how many of these attributes the users consider, which of them the users focus on, whether they are used in the decision process with each other or separately, and their relation to the overall rating.

\section{A. Users Surveys}

In order to understand how users perceive reputation through ratings and reviews, we have conducted a survey where the participants were asked a series of questions. [1] The study ran for three weeks with 200 participants. The description, objectives, and results of the survey are

Objectives: The survey objectives were:

- show that users use more information than the usual stars and reviews in their buying decision

- compare between ratings representation

- prove that detailed-ratings style confirms with the social notion more than single-ratings style

- discover what reputation information the users actually use to decide on a seller (i.e. attributes the users use in their decision making) 
- examine the participants' perception and interpretation of online ratings

- importance of detailed textual reviews

- frequency and size of cooperation between users

We extended the study by interviewing several users. In these interviews the users were split into two groups. The first group was asked about current rating and reviewing systems. The second were given new forms of ratings based on multiple categories and tested for the maximum amount of rating attributes that they would like to rate which was 4 attributes in average..

Results: The results confirmed the following:

1) $60 \%$ of the users who were given the choice between an e-Bay rating style (i.e. 563 and a gold star) and a detailed rating without the number of reviewers (i.e. ratings for price, delivery, quality), preferred the detailed rating.

2) When the users were asked to choose the interpretation of different representations (see figure 1) where there was no "reviews" option available, they explicitly asked for it to be added stating that this is the only way to gather more information on a provider before selecting him.

3) In a comparison between ratings representations, stars was the most liked, percentage was the most trusted, most expressive, and the most understandable. The results are shown in figure 1.

4) When the users were asked about how often they rate on the internet, $65 \%$ answered sometimes and $21 \%$ answered never.

5) When presented with examples of amazon ratings, $80 \%$ of the participants considered the rating is for the quality of the product, $20 \%$ did not know what it represents.

6) When presented with the choice between a seller with higher stars values (i.e. 4 stars vs. 3 stars) and a seller with less star value but higher number of reviews, $80 \%$ of the participants considered number of reviews more important (i.e. selected the seller with more number of reviews). When presented with the option between high number of reviews versus detailed ratings, $60 \%$ chose detailed ratings.

7) According to the results from this set of participants, the entities that they rate the most on the internet are products from e-shops.

8) The users were asked a question of how they immediately interpret the statement "high seller reputation", $50 \%$ of the participants consider a high or a good seller reputation is a conjoint measure of price and quality, while $39 \%$ interprets it as good customer service.

Analysis: From the aforementioned results, we can conclude that there is a confusion in interpreting not only the meaning of rating styles but also the meaning of reputation values. Reputation of a seller or a product means more than one attribute i.e. product quality or seller customer service.
It is usually perceived as a combination of attributes that are neither represented nor clear from current rating methods (results 2,5,8). Detailed ratings (though simple in our examples here) were preferred over stars ratings and number of reviews (results 1,2,6). Furthermore, it is clear that users tend to read reviews (i.e. textual comments and feedback) in order to decide on a product or a seller. This means that the singular formats of reputation is not enough and review analysis is required to acquire more information on the entity being rated. This is possible for human users though time consuming and sometime a tedious task. But in other domains such as the reputation of software agents or web services this is not possible. Text analysis is a highly expensive task that can not be performed for every transaction.

\section{B. Online Chocolate Store}

Online stores became a vital part of the internet during the last decade. Research on design and user interactions focused on how to both review and promote the products being sold. Recommendation systems focused on how to bring to user attention products -or services- that may interest him/her. Large e-commerce companies such as Amazon and e-Bay are also interested in enhancing the buying experience for their users. For such companies, it is critical to know the reasons for their customers choices. One of the most important factors that affect user behavior when buying a product is product reviews and ratings [11].

Based on the study explained in III-A, we have decided to conduct an experiment that shed some light on user behavior regarding multiple or categorized rating versus overall rating. In this experiment we sat up an actual online store selling chocolate for our institute personal only (for legal reasons). The ChocStore was implemented with an online store basic functionalities i.e. browsing products, choosing products, putting them on a shopping basket, and placing an order. Users were asked for their institute email address for authorization, a cover-flow of nine different chocolates is shown, the user chooses the chocolate and places them in the basket, then the user is directed to an order page and is asked for the delivery method which varied from office delivery or delivery in a box in the reception area where the user had to pick it up within a certain amount of time. The user then is also given two choices of payment and directed to conform the order. This is followed by an email conforming the order and predicting when it will be delivered.

Objectives: The objectives of the experiment were

- to observe how users interact with the system,

- how many attributes are suitable for a review and the time it takes to fill in multiple ratings form,

- what is the important aspect of each user's rating,

- to prove that single rating is not enough by showing that several attributes - delivery time for instance- affects rating, and

- to examine categorized ratings with multiple attributes versus overall ratings. 


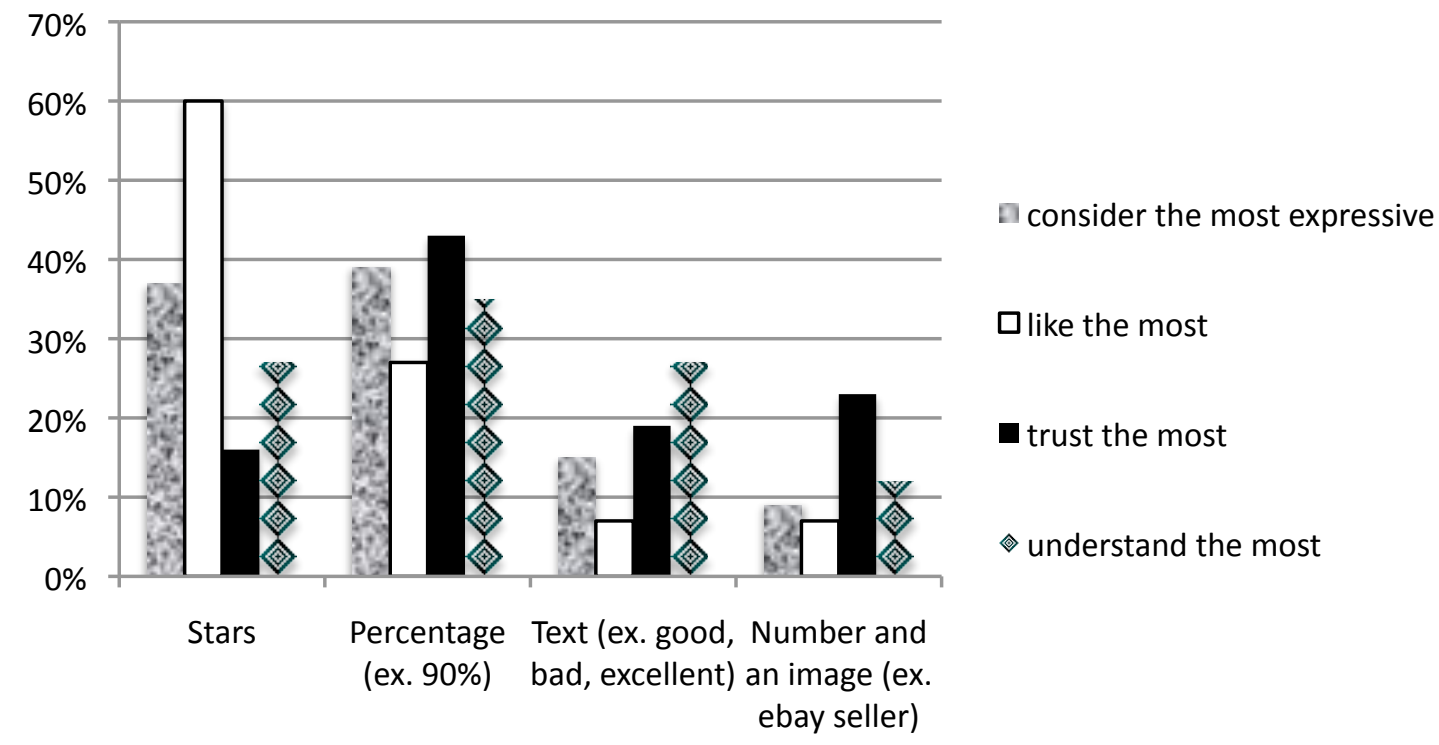

Fig. 1. Comparison between ratings representations based on survey results

For the last two objectives, we setup the final rating form to have both categorized ratings and an overall rating.

To ensure impartial decisions from the users, the chocolate was displayed in a random order and we offered three different brands each of which has three different kinds. Moreover, the prices were changed significantly compared to the procurement cost (i.e. some were underpriced and others were overpriced). The implementation of the store was part of a master thesis supervised by the authors [9] on recommendation systems.

Once the order is delivered, the user is asked to rate the whole buying experience including the speed of delivery, the convenience of the payment method, the quality of the products, the price of the products, the overall buying experience, and the crucial aspect of his/her rating (figure 2). That conforms to three general categories: payment method, delivery method, and product that has two attributes/subcategories of quality and price. Thirty six orders have been received with a total of eighteen users participating in the experiment. Some of these orders have been deliberately delayed in delivery.

Results: Results showed that:

1) There was a negative correlation between price and sales; the lower the price the higher the sales.

2) The overall rating does not always relate to the same attribute (i.e. delivery, quality, price), therefore, overall rating does not convey or show the meaning behind it.

3) Delivery time affects delivery rating and sometimes overall rating.

4) Prices always affect overall rating.

5) When the users where asked (see fig. 2) which attribute they based their overall rating on (i.e. to pick one attribute that is most important to their rating), $38.5 \%$ was on price, $38.5 \%$ was on quality, and $23 \%$ was on delivery.

Comparing the overall rating (which varies from 2 for low rating to 4 for high rating) versus each attribute rating, the observations were:

- users who gave an overall rating of 4 can be divided into 3 groups: the first gave high ratings for product quality and prices and did not care about the delivery. The second group gave high rating for delivery and did not care about the price or the quality. The third group gave high ratings for all attributes.

- users who gave an overall rating of 3 can be divided into 2 groups: the first gave this rating because of product quality and the second group because of product delivery

- users who gave an overall rating of 2 was for late delivery and were neutral on product quality and price

Analysis: The results show that users gave the same overall rating for different reasons. Some cared more for the product quality and others cared for how fast the delivery is. For each group of users, the preferences used in their buying decision were different. Therefore, an overall rating is not enough to evaluate the reputation of a service provider and it certainly does not give other users the choice for customized selection (i.e. based on one of the aforementioned attributes). According to result no. 5, the number of participants who based their ratings on price is the same as the number of participants who based it on delivery. This means that a rating value $x$ given by a user because of good quality does not have the same meaning of a rating value $x$ given by a user because of price. Furthermore, we asked all participants about their satisfaction of the rating form -regarding the number of rating attributes- and all of them were satisfied with the form noting that what they do not like is taking the time to write long 


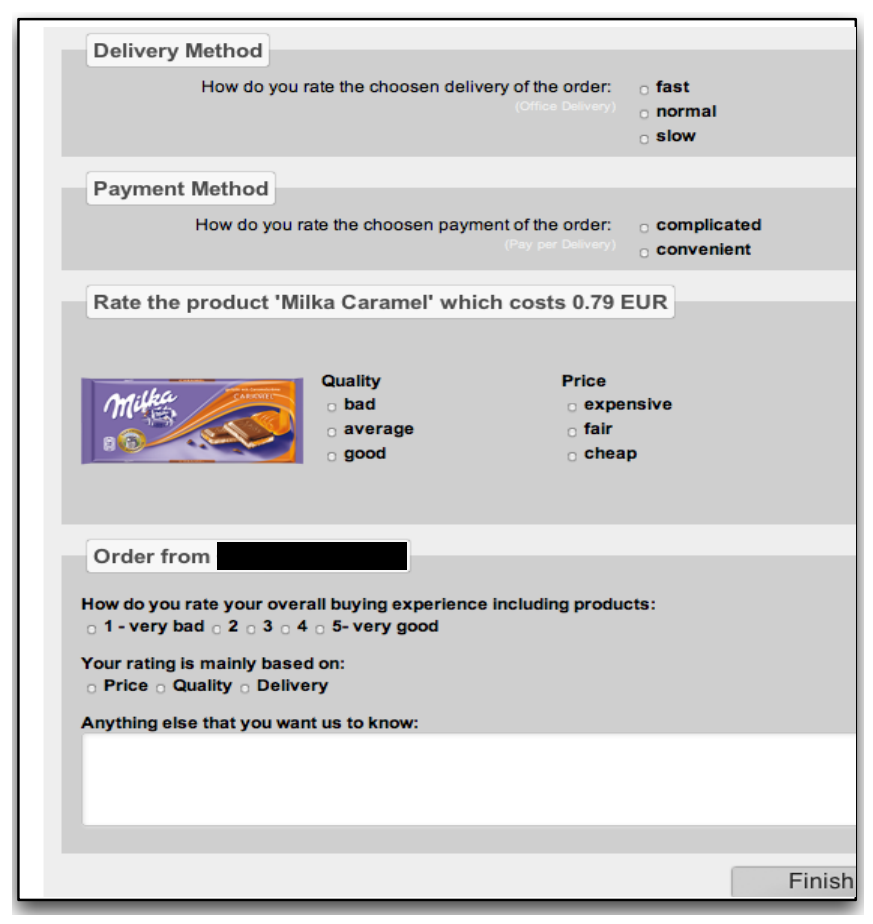

Fig. 2. ChocStore Rating Form

reviews. The form gave them the opportunity to express their perception of rating on different levels.

Since the ChocStore was designed as a single provider online store, it was not possible to conduct a study on user choices of providers based on the presented categorized ratings.

\section{Discussion}

Open collaboration poses a challenge of what is being shared, how it is being shared, and the common versus the intended meaning of this information. Users use several pieces of information to decide on a service provider. Their reasons for satisfaction/dissatisfaction or for deciding on a service provider differ from one another. Single format ratings tend to ignore the reasons and information behind the ratings. These ratings are used to construct the reputation of a service provider despite the lack of enough semantic information for the reasons behind them. A user seeking a provider checks for high reputed ones assuming that the high reputation interprets into his own attribute of selection. A more aware user has to read textual reviews for the service providers on the domain in order to find what he/she is looking for. This is more suitable for online reputation systems with user interfaces to transmit such user experience but it is not suitable for other domains where automated software agents have to make the same decision. Products or services have to be described consistently in terms of attributes and levels in order to see what is being traded off and what is actually being rated. During the aforementioned studies, we were able to confirm the two hypothesis explained in the introduction.
Based on the study discussed in section III-B and other studies we conducted on other domains [15][4], we have developed an ontology to represent reputation in a format that is open, interoperable, and embedding reputation knowledge [6]. We have used the data from the chocStore to test our ontology and reputation knowledge transfer.

\section{CONClusion}

Reputation systems depend mainly on users sharing their experiences and opinions in a certain domain. The nature of the information being shared depends on the user perspective. In this paper, we explained our hypothesis that the users usually use more reputation information than the one being represented in current formats and that these formats are not enough to convey an entity's reputation. We examined the hypothesis via two user studies and showed the results that confirmed them. We also explained the problems with current reputation systems along with the general steps to construct one. In future work, we plan to examine whether we can predict user buying decision based on our model in [6].

\section{REFERENCES}

[1] Online survey on rating systems. http://www.kwiksurveys.com?s= ILNING_865460b6.

[2] Alfarez A bdul-Rahman and Stephen Hailes. Supporting trust in virtual communities. In Proceedings of the 33rd Hawaii International Conference on System Sciences-Vol. 6. IEEE Computer Society, 2000.

[3] Rehab Alnemr, Justus Bross, and Christoph Meinel. Constructing a context-aware service-oriented reputation model using attention allocation points. Proceedings of the IEEE International Conference on Service Computing, 2009.

[4] Rehab Alnemr, Stefan König, T. Eymann, and C. Meinel. Enabling usage control through reputation objects: A discussion on e-commerce and the internet of services environments. In Special issue of Trust and Trust Management, Journal of Theoretical and Applied Electronic Commerce Research, 2010.

[5] Rehab Alnemr and Christoph Meinel. From reputation models and systems to reputation ontologies. In 5th IFIP WG 11.11 International Conference on Trust Management (IFIPTM), 2011.

[6] Rehab Alnemr, Adrian Paschke, and Christoph Meinel. Enabling reputation interoperability through semantic technologies. In $A C M$ International Conference on Semantic Systems. ACM, 2010.

[7] Rehab Alnemr, Matthias Quasthoff, and Christoph Meinel. Taking Trust Management to the Next Level. Handbook of Research on P2P and Grid Systems for Service-Oriented Computing: Models, Methodologies and Applications, pp. 796-816, 2009.

[8] John M. T. Balmer and Stephen A. Greyser, editors. Revealing the corporation. Routledge, London [u.a.], 2003.

[9] Manuel Blechschmidt. An architecture for evaluating recommender systems in real world scenarios, 2011.

[10] J. Chandler, K. El-Khatib, M. Benyoucef, G. Bochmann, and C Adams. Legal challenges of online reputation systems. In In L. K. R. Song, Chapter in Trust in E-Services: Technologies, Practices and Challenges, pages 84-111. Hershy: Idea Group Publishing, 2007.

[11] Anindya Ghose and Panagiotis G. Ipeirotis. Designing novel review ranking systems: Predicting usefulness and impact of reviews, 2007.

[12] Audun Jøsang, R. Ismail, and C. Boyd. A survey of trust and reputation systems for online service provision. Decision Support Systems, pages 618-644, 2007.

[13] Robert Kosara, Christopher G. Healey, Victoria Interrante, David H. Laidlaw, and Colin Ware. User studies: Why, how, and when? IEEE Computer Graphics and Applications, 23(4):20-25, 2003.

[14] Jordi Sabater and Carles Sierra. Review on computational trust and reputation models. Artif. Intell. Rev., 24:33-60, September 2005.

[15] Maxim Schnjakin, Rehab Alnemr, and C. Meinel. A security and highavailability layer for cloud storage. In The 2nd Int. Workshop on Cloud Information System Engineering (Springer CISE 2010), 2010. 